\title{
Research on Novel Natural Image Reconstruction and Representation Algorithm based on Clustering and Modified Neural Network
}

\author{
LU Dong-xing ${ }^{1}$ \\ ${ }^{1}$ College of Electronic Information and Mechatronic Engineering,ZhaoQing \\ University,Zhaoqing,526061,China
}

\begin{abstract}
In this paper, we conduct research on the novel natural image reconstruction and representation algorithm based on clustering and modified neural network. Image resolution enhancement is one of the earliest researches of single image interpolation. Although the traditional interpolation and method for single image amplification is effect, but did not provide more useful information. Our method combines the neural network and the clustering approach. The experiment shows that our method performs well and satisfactory.
\end{abstract}

Keywords: Natural Image; Clustering Method; Modified Neural Network; Image Representation.

\section{Introduction}

For a natural image which includes both edge and texture information, the single basis function cannot reconstruct the image for compressed sensing optimally. Compressed sensing theory of the core idea is to unite the sparse sex prior knowledge of signal in the process of signal reconstruction from far less than the sampling rate of sampling points to reconstruct the original signal, thus effectively reduce the complexity of the sensor and the sampling system. In the traditional data acquisition system, the Shannon sampling theorem is must follow the basic principles, namely the signal sampling frequency must be greater than or equal to two times the bandwidth of the signal to be able to reconstruct the original signal without distortion. In broadband large capacity data acquisition system, in order to save storage capacity and transmission bandwidth, often start with higher than or equal to the sampling rate of the sampling rate to obtain the original data, and then to a sparse transform of image, abandons the transform coefficient of small reserves significantly transform coefficient, and then to encode the transform coefficient significantly after storage or sent to the channel transmission. Synergy neural network is to construct the network by means of top-down, its biggest characteristic is that there is no false state, and the emergence of the pseudo state met the biggest difficulty has been the traditional neural network [1-4].

Image resolution enhancement is one of the earliest researches of single image interpolation. Although the traditional interpolation method for single image amplification is effect, but did not provide more useful information, so didn't actually improve the resolution of the image. According to the theory of signal reconstruction, the use of image sequence to provide related and complementary information and prior knowledge can better recovery and reconstruction of the original image details. Super-resolution image reconstruction is to point to by a picture or a series of low resolution degradation image or to generate a series of high resolution image of the process, but also can eliminate noise and produced by finite detector and optical components such as fuzzy image degradation. In the compressed sensing system, the sparse representation of image is a key factor affecting the quality of image reconstruction. At present in 
the compressed sensing image reconstruction using only a single image. The basic functions but most natural images consisting of smooth, edge and texture at the same time three ingredients are just different proportion of these three components. Single basis function cannot at the same time provide optimal said on three components. Based on a priori Huber-Markov random field of maximum a posteriori probability estimation method, improve the quality of the reconstructed image and this method is relatively complex, large amount of calculation. In recent years, the study of partial differential equation for image rich and mature gradually, is widely used in image processing and computer vision which has developed into a rigorous in theory, on the practical effective method. This paper will be introduced to the method of partial differential equation for image super-resolution image reconstruction, by using the nonlinear diffusion model as a robust and effective method is put forward.

To enhance the current existing algorithms, we conduct theoretical analysis and experimental practice on the topic of novel natural image reconstruction and representation algorithm based on clustering and modified neural network. Purpose is to develop the fusion effect is good, image details outstanding fusion method for the practical application of the infrared and visible light image fusion provides a new way. In wavelet transform domain, the low frequency approximation and the average characteristics of the image reflects the original image, the high frequency sub-image of reflecting the mutation characteristics of original image, the image edge features and regional boundary. In the following sections, we will discuss the issues in detail. In the figure one, we illustrate the sample dataset for simulation which is obtained from Harvard University.
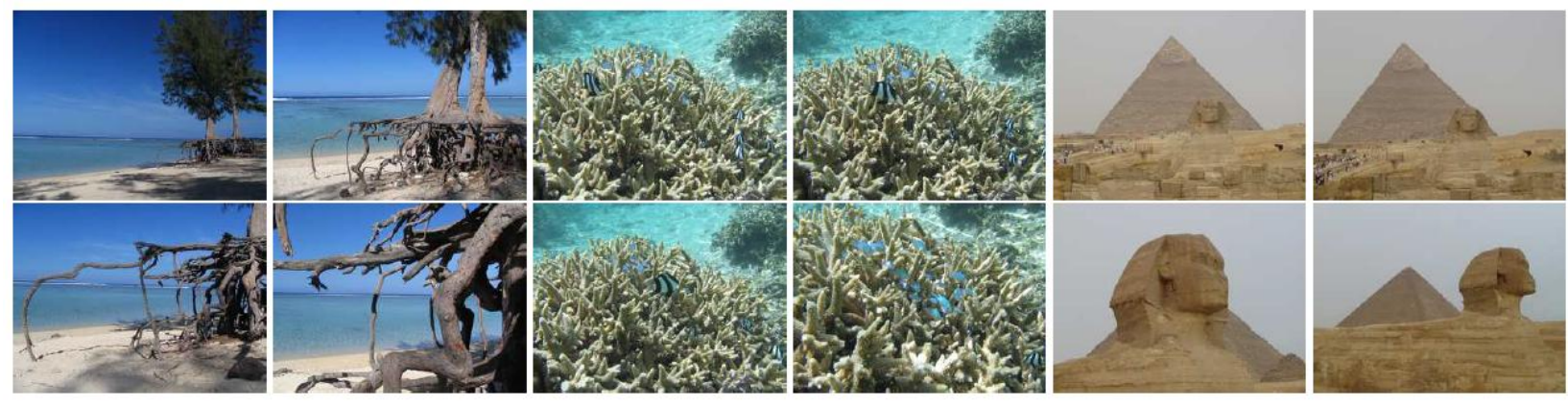

Fig. 1The Dataset used for Analysis and Simulation

\section{The Theoretical Analysis on Novel Algorithm}

The Descriptive Image Reconstruction. Image super-resolution reconstruction technology and some other image processing techniques such as image de-noising, image to fuzzy, image restoration, image enhancement, image interpolation and closely related to image fusion, however these relationships in the summary of the literature has not been system. Image fusion is to use an algorithm to combine two or more information of the image to generate a new image of technology. From this definition it can be seen that image fusion than super-resolution image reconstruction has a broader meaning, namely the image fusion can include image super-resolution reconstruction. Based on a particular observation model of image de-noising and image to fuzzy can be referred to as the image restoration. As a result of traditional image restoration which can only to restore blur and noise etc., after processing pixels did not increase, so cannot be regarded as the image super-resolution reconstruction technology. But in fact, the image restoration and super-resolution reconstruction is related field, a 
lot of super-resolution reconstruction methods are introduced from single image restoration, so the super-resolution reconstruction of images can be seen as the second generation recovery. In the formula one, we show the general model description.

$$
V_{i}=\left(v_{i, 1}, v_{i, 2}, \ldots, v_{i, c}\right)
$$

Image enhancement technique is the most basic image processing techniques, the purpose is for image processing, highlight the image of certain information and weaken or eliminate some unnecessary information, in order to get better visual effect for specific application, more useful images, or converted to a more suitable person or a machine is analyzed in the form of processing.

$$
\min _{\alpha} \frac{1}{2}\|\alpha\|_{2}^{2}+C \frac{1}{N} \sum_{i=1}^{N} \Delta\left(f\left(X_{i}\right), y_{i}\right)
$$

(2)

Image enhancement and image restoration (including super-resolution reconstruction) in the cross area, but the image enhancement is mainly a subjective, the exploratory process, is a characteristic of the physiology of human visual system to accept and to design a way to improve the image. Most of image restoration and super-resolution reconstruction is an objective process which is trying to use the degradation phenomenon of some prior knowledge to recuperate the degraded image, namely the recovery or reconstruction technique is to put the degradation model. In the following figure two, we show the descriptive image structure and organization result.
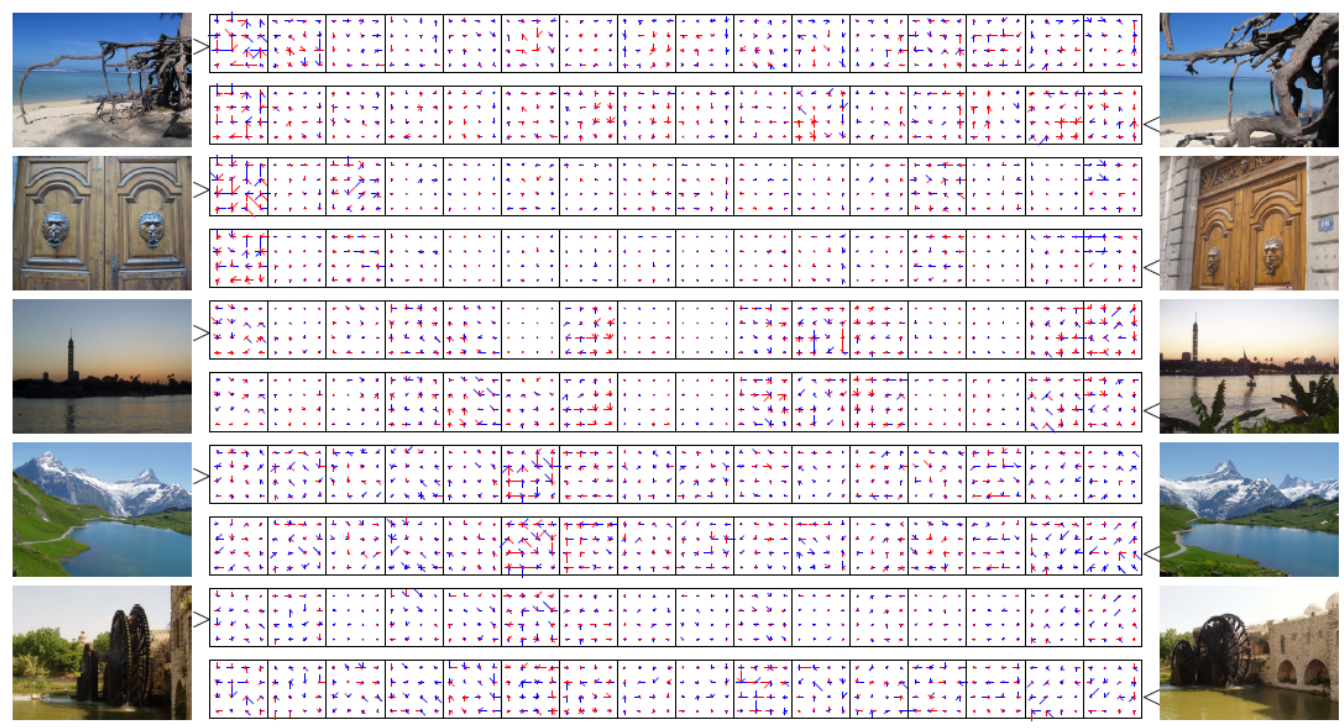

Fig. 2The Descriptive Image Structure and Organization Result

The Modified Neural Network. Multi-layer perceptron network structure, for example, the entire network is an inseparable whole, between each neuron function, to study and deal with the data of the same or similar to each other, and basic function is to set up network mapping from input to output, unable or difficult to differentiate between input and output process of gradual transformation. Stereotypes neural network can be layered structure step by step, the basic function of the network divided into several independent functional modules. Algorithm based on search mechanism is the most representative in the former BP learning algorithm and simulated annealing algorithm to the network. The BP algorithm is essentially a network error sum of squares of the objective function, according to the gradient method and 
its algorithm to minimize the objective function value. Its theoretical basis, the physical concept is clear, made it possible to get all kinds of feed forward neural networks application. The formula three shows the detail function.

$$
\max _{x} \min _{k}\{|x, c|\}, i=1,2,3, \ldots, m
$$

Because gradient method with only local search ability, if used to have multiple minimum point of the objective function, the drawback of the symbol is unable to avoid falling into local minimum, so the algorithm is not complete. In order to overcome the shortcoming of BP algorithm easy to fall into local minimum, people inspired from the annealing phenomenon, introduce the simulated annealing algorithm. In order not to fall into local minimum, in the process of using gradient method for iteration, cannot completely according to the direction of the gradient descent iterations, but given a chance to small probability, according to the different directions iterate. For similar samples border area many test samples may not be reject, the covering algorithm was improved, determine the spherical field, the field was reduced, become contains only the minimum cover this kind of training sample. However, is not the same sample ask the boundary of the large area, when the test sample into this area, the category of belonging is difficult to determine.

Coverage algorithm with the hyper-plane of cutting sphere and spherical field of neuron structure neural network, classification ability, running speed, can realize the division of multidisciplinary problem at the same time. But it is suitable for the more problem of linear separable, for nonlinear separable problem more efficiency is not high. Rough set method can be well about attribute reduction of redundant information, extract the minimum core attribute set, effectively reduce the attribute dimension, and according to the non-numeric attributes in rough classification, would be in the first part of the subset of the user intent, regardless of the remaining subset, thereby reducing the number of samples of subsequent coverage algorithm. Large-scale pattern recognition problem in particular category is difficult to solve the problem of pattern recognition. This kind of problem which is the feature of high dimension space, large sample size and the number of categories involved.

The Combined Novel Representation Methodology. In order to reduce the consumption of memory, the most direct way is to put the image is divided into a certain size and number of image block, processing for each image block processed separately, and then the processed image stitching together. Combined the method of solving are generally based on maximum a posteriori estimation or maximum likelihood comes up framework reconstruction model is set up. When image size is not large, sparse matrix of image storage, and using appropriate mathematical accelerated when solving strategy can greatly improve the speed of super-resolution reconstruction. Chunking arithmetic method is mainly by reducing the dimensions of the coefficient matrix to get rid of the limit of memory, so as to improve the speed of operation. Super-resolution reconstruction of another way of operation is directly with the method of image operator, namely in the process of operation does not need to request a specific form of the matrix. In the process of solving the matrix and vector multiplication by using the following method: the vector is regarded as an image, multiplication can be seen as the result of the operator on the result of image processing. Block operation, however, ignore the continuity and correlation between block and block and the processing result often is not optimal. In the figure three, we show the performance of the proposed algorithm. 


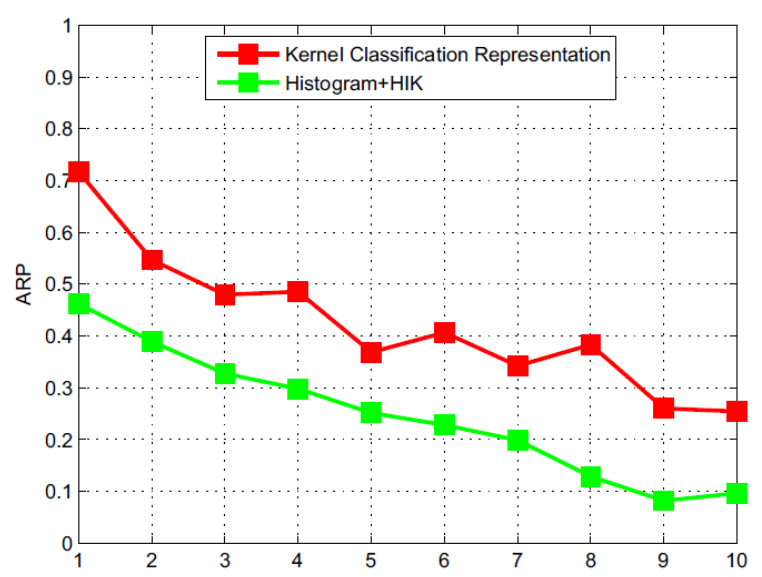

Fig. 3The Performance of the Proposed

Algorithm

\section{CONCLUSIONS}

This paper discusses a theoretical analysis and experimental practice on the topic of novel natural image reconstruction and representation algorithm based on clustering and modified neural network. In broadband large capacity data acquisition system, in order to save storage capacity and transmission bandwidth, often start with higher than or equal to the sampling rate of the sampling rate to obtain the original data, and then to a sparse transform of image. When image size is not large, sparse matrix of image storage, and using appropriate mathematical accelerated when solving strategy can greatly improve the speed of super-resolution reconstruction. The experimental result illustrates the effectiveness and robustness of the proposed methodology.

\section{References}

[1] Movshon, J. Anthony, and Eero P. Simoncelli. "Representation of naturalistic image structure in the primate visual cortex." Cold Spring Harbor symposia on quantitative biology.

[2] Rahman, Md Mahmudur. "A soft image representation approach by exploiting local neighborhood structure of self-organizing map (SOM)." Soft Computing (2015): 1-11.

[3] H. Wang and J. Wang, "An effective image representation method using kernel classification," in Tools with Artificial Intelligence (ICTAI), 2014 IEEE 26th International Conference on, Nov 2014.

[4] Chen, Jiann-Jong, et al. "Matrix Variate Distribution-Induced Sparse Representation for Robust Image Classification." (2015). 